Aperture judgement in fire-appliance drivers

\author{
Victoria Kroll and David Crundall \\ Department of Psychology \\ Nottingham Trent University
}

This is a post-print version of the following article:

Kroll, V., and Crundall, D. (2019). Aperture judgement in fire-appliance drivers. Transportation Research Part F. Forthcoming.

Address for correspondence:

Prof. David Crundall

Department of Psychology

Nottingham Trent University

David.crundall@ntu.ac.uk

+44(0) 1158482358

Keywords: Fire and Rescue Service, fire-appliance driving, aperture judgement, driver safety, eye movements 


\title{
Aperture judgement in fire-appliance drivers
}

\begin{abstract}
Low-speed collisions are a common occurrence for UK Fire and Rescue Services, with frequent bumps and scrapes adding up to create a significant drain on emergency service budgets, and damaging public faith in the service. We hypothesised that the decision to drive through a gap, or aperture, created by other vehicles and/or road furniture, may be related to driver experience and risk-level. To create a test to assess this skill, video clips were recorded from a fire appliance on bluelight training runs. Eighteen clips were selected on the basis that they culminated in a narrow aperture that the film-driver either navigated through, or stopped and waited for other vehicles to move and the gap to widen. Drivers were required to watch these clips, which paused at the point that the film-driver made the decision to either go though, or to wait. The participants rated their belief that the appliance would fit through the gap, and how confident they would be to drive through that aperture themselves (on a 1-8 scale). Novice fire-appliance drivers were less accurate in deciding whether to proceed or not, though their criterion threshold did not differ to that of more experienced drivers. All drivers favoured looking at the right edge of the aperture (either a vehicle or road furniture), but low-risk, experienced fire appliance drivers had the shortest fixations on this area. The results suggest that a video-based test of aperture judgement can differentiate between fire-appliance driver groups based on experience, opening the way for future testing and training tools using this methodology.
\end{abstract}




\section{Introduction}

The ability to manoeuvre through the physical environment without colliding into obstacles is a fundamental skill. Often obstacles define the space through which we need to navigate, creating 'apertures' or 'gaps', such as a doorway if traveling on foot, or a gap defined by other vehicles and street furniture if travelling in a car (Wilmut, Du, \& Barnett, 2015). In such situations, one must judge whether they, or their vehicle, will fit through the gap without collision. When walking, if the gap is perceived to be too narrow for the body to fit through (e.g. the gap between on-coming pedestrians) then the individual can make a postural adjustment by rotating their body in the yaw dimension to reduce the horizontal space necessary for them to pass through (Warren \& Whang 1987; Higuchi et al. 2006, 2011, 2012; Cowie et al., 2010; Franchak \& Adolph 2012; Franchak \& Adolph, 2014; Hackney et al. 2014, 2015a, b; Muroi \& Higuchi, 2017). These postural adjustments can be achieved at extremely short notice. However, when driving, one cannot simply rotate the vehicle to navigate a gap. Instead, the driver must make a relatively early decision as to whether the aperture is sufficient for the vehicle to fit through. If an aperture is not sufficient, and at least one edge of the aperture is defined by another vehicle, the driver must wait for the other vehicle to move before proceeding.

This problem is further exacerbated when travelling in a particularly large vehicle, such as a fireappliance (FA hereafter). FAs vary in size, but typically weigh over 12 tonnes and require an HGV licence to drive. Despite the size of such vehicles, drivers must often drive above the prevailing speed of nearby traffic, and may need to contravene typical road rules to get to a reported incident as quickly as possible. We have recently developed a hazard prediction test, specific to FA drivers, that assesses their ability to spot serious potential collisions (Crundall and Kroll, 2018). However, the biggest drain on fire service resources are not the serious, yet infrequent, collisions, but the relatively innocuous low-speed crashes that occur on a more frequent basis. For example, many collisions occur with street furniture, fire-station buildings, or other road users while trying to squeeze the FA through a gap or aperture that is too small. While such collisions might only result in damaged side-mirrors, or minor scratches or dents, the cumulative cost poses a significant problem for fire services operating on restrictive budgets.

Typical studies of gap judgement, or gap acceptance, are generally focused on situations where a car driver is trying to enter or exit a junction. A gap in this context is defined as the space headway or time between two successive vehicles in a stream of traffic (Karthika \& Koshi, 2014; Obdaidat \& Elayan, 2013). While this definition of a gap differs to our current focus in this paper, a brief review of such studies may be beneficial.

In these traditional gap-judgement studies, the driver must evaluate the gaps in the flow of traffic and judge whether they are sufficient to allow a safe manoeuvre, whilst at the same time controlling the spatiotemporal characteristics of the vehicle to avoid colliding with incoming traffic (Karthika \& Koshi, 2014). Incorrect judgements are likely to underlie a significant number of junction collisions (Baures, Oberfeld, Tournier, Hecht \& Cavallo, 2013). Such decision-making processes are typically studied by presenting videos or simulated situations of approaching vehicles (cars, lorries, motorcyclists and trains), and participants are required to respond when a vehicle has reached a specific location (e.g., Berthelon \& Mestre, 1993; Caird \& Hancock, 1994), or when they believe it is safe to pull out from, or into, a side-road (e.g. Crundall, Crundall, Clark and Shahar, 2012; Dewing, Duley, \& Hancock, 1993; Hancock, Caird, Shekhar, \& Vercruyssen, 1991; Louveton, et al., 2012)

Previous studies using this methodology have revealed that drivers accept different gap sizes depending on the size of the approaching vehicle, with the larger vehicles being judged as arriving earlier than smaller vehicles travelling at the same speed (Caird \& Hancock, 1994; Horswill et al., 2005; Bottom \& Ashworth, 1978; Keskinen et al., 1998; Alexander et al., 2002). This size arrival effect was 
first identified with simple geometric shapes (DeLucia, 1991), and has been a commonly cited explanation for this pattern of behaviour in time-to-arrival studies (Lee \& Sheppard, 2017). For instance, the size-arrival effect can explain why cars are often judged to arrive earlier at a junction than motorcyclists (e.g., Hancock \& Caird, 1993; Hancock, Caird, \& Johnson, 1991; Nagayama, Morita, Miura, Watanabe, \& Murakami, 1980).

As with many driving skills (e.g., hazard perception), the ability to accurately judge a gap in traffic develops with driving experience and exposure. For example, research suggests that novice drivers with less than three years of driving experience have a greater difficultly with gap acceptance than experienced drivers (Clarke, 2000; Crinson \& Grayson, 2005; Forsyth, 1995). This may explain their over-representation in collision statistics when turning right at a junction (Clarke, Forsyth, \& Wright, 1998; Forsyth, 1995; West et al., 1993). Differences in drivers' eye movements may partially underlie this experiential effect, as experienced drivers tend to have a wider and more varied search pattern than novice drivers at junctions (Scott, Hall, Litchfield \& Westwood, 2013). A similar finding was observed by Crundall et al. (2012), who found that novices did not search as far down the road as more experienced drivers when deciding whether it was safe to pull out.

Though the above literature provides important points for the current research (namely the role of experience and eye movements in the accuracy of gap judgements), the method does not suit the present problem under investigation. While gap acceptance when pulling out of a junction remains relevant to emergency service drivers, the current focus is more akin to the navigation of apertures that is found predominantly in the walking literature (e.g., Warren \& Whang 1987; Higuchi et al. 2006, 2011, 2012; Cowie et al., 2010; Franchak \& Adolph 2012; Franchak \& Adolph, 2014; Hackney et al. 2014, 2015a, b; Muroi \& Higuchi, 2017). Specifically, following discussions with fire service representatives, we decided that a more pertinent test of gap judgement would be to assess a driver's ability to judge whether the FA can fit through an aperture bounded by two static objects (e.g. a car that has pulled over, and road furniture). This scenario is extremely common: When average car drivers are faced with an approaching FA from behind they will typically pull over to make space for the FA to progress. While drivers do this with the best intentions, many often fail to consider the extent of the passing space that they are leaving for the FA. Some drivers may pull over opposite other stationary vehicles, or close to road furniture, such as central reservations. This creates a bottleneck through the which the FA driver must travel. Although the FA driver will reduce speed these points, an incorrect decision to progress through a gap that is too narrow, will result in low-level damage to the FA, and other vehicles or road infrastructure.

To the knowledge of the authors, there are no journal articles that refer to driver skill in navigating cars through such apertures. The closest field of research is in the use of Urban Search and Rescue robots: Schmidlin and Jones (2016) found tele-operators to improve with repeated exposure to aperture decisions (with feedback), which suggests that this skill is likely to improve with domain experience. Accordingly, we designed a test to assess fire-appliance drivers' ability to accurately judge whether their vehicle would fit through a particular aperture. Such a test, if successful, could act as an assessment tool for FA drivers, or, when coupled with appropriate feedback, could provide the basis for a training intervention.

A multi-camera system was placed on a fire appliance during pre-planned blue-light training runs around Nottinghamshire. The multi-camera system allowed us to capture all the visual information that is available to the driver of the FA. This resulted in over 12 hours' worth of video footage, from which 18 clips ( 9 'go-trials' and 9 'no-go trials') were selected to create the aperture-judgement test. In all trials, the FA was on blue lights and the end of each clip the FA was presented with a naturallyoccurring aperture, typically caused by other vehicles stopping at inappropriate locations to let the FA pass. Such gaps were typically between two vehicles, or between a vehicle and road furniture. Participants were required to answer two questions in terms of (1) how likely they thought that the 
appliance could fit through the gap and (2) how confident they would be as the driver at getting the appliance through the gap unscathed. Both questions required participants to give a response on a 1 -8 Likert scale.

We recruited four different groups of drivers: a control group of non-emergency response drivers and three groups of FA drivers: novice drivers, high-risk, experienced drivers and low-risk, experienced drivers (based on the number, severity and blameworthiness of self-reported incidents). The comparisons of the different driver groups were intended to reflect different experimental hypotheses. First, all FA drivers were compared to control drivers. Even our novice FA drivers are highly experienced car drivers, and have trained for an HGV licence. As such, we expect that all our emergency service drivers should have superior performance compared to average car drivers (cf. Crundall et al., 2003, 2005; Horswill et al., 2013; Johnston \& Scialfa, 2016; McKenna \& Crick, 1991).

Second, we compared experienced FA drivers (both high- and low-risk) to novice FA drivers, on the assumption that the latter might perform worse (cf. Clarke, 2000; Crinson \& Grayson, 2005; Forsyth, 1995; Clarke et al., 1998; Forsyth, 1995; West \& French, 1993; Crundall et al., 2012). There are also theoretical reasons for believing that novice FA drivers will be less accurate at judging whether an appliance can fit through a specific gap. Peripersonal space (PPS) refers to the space around one's body that essentially falls within arm's length. This area of the world has a specific area of the brain assigned to it, which controls the representations of all things that we can immediately interact with (i.e. reach out and grab). Many studies have however demonstrated that tool use (e.g. using a stick to touch objects that are outside arm's reach) extends peripersonal space (e.g. Holmes et al., 2004). Indeed, a recent study demonstrated that a wheelchair can be considered as a 'full-body' tool which extends the representation of PPS in individuals following training (Galli et al., 2015). It is a logical extension of these findings to suggest that a vehicle may extend the driver's PPS in a similar fashion (Ho \& Spence, 2008). In other words, an experienced FA driver may almost feel that the vehicle is an extension of their own body. This integration of the fire appliance with the driver's body schema would be just one possible route to more accurate aperture judgements with increased vehicle-specific experience.

Finally, we compared high- and low-risk groups of experienced FA drivers to examine whether the test could discriminate between safe and less-safe drivers. Given that the majority of FA collisions happen at slow speed when doing manoeuvres, this would make logical sense as less-safe drivers, based on previous collision history, presumably make more aperture-judgement errors. 


\section{METHOD}

\section{Participants}

Eighty-four drivers were recruited and assigned across four driver groups. The first group consisted of 21 novice FA drivers ( 3 female) with a mean age of 35.4 years and a mean personal driving experience of 16.5 years since passing their driving test. Forty-three experienced FA drivers were split into high risk (20 in total, all male) versus low risk ( 23 in total, 2 female) on the basis of self-reported frequency, severity and blameworthiness of all recalled collisions across their driving history (including personal and at-work collisions). Severity ratings for each collision varied between 1 and 3 points, based on damage and injury. Blame ratings also varied between 1 and 3 points, with increasing points reflecting greater responsibility on the part of our drivers. The mean number of reported collisions were 0.56 and 2.85 for low and high-risk groups, with mean summed severity/blame scores of 1.7 and 10.7, respectively. Please refer to Crundall \& Kroll, 2018 for further details. These participants had a mean age of 42 , a mean of 10.4 years' experience of fire-appliance driving, and a mean driving-experience of 23.4 years. The final group consisted of 21 control drivers ( 20 in total: 1 female), with a mean age of 43.9 years and a mean personal driving experience of 22 years, none of these participants had a HGV license.

\section{Materials and apparatus}

\section{Filming}

The filming took place over a period of 4 weeks from April to May 2015. For the filming, seven cameras were attached to a fire-appliance during scheduled blue-light training runs. Training runs were used to avoid undertaking unnecessary non-emergency blue-light runs beyond those needed for training purposes. This resulted in a total of approximately 12 hours of footage. For more details of the filming of the footage please see Crundall and Kroll (2018).

\section{Editing and Clip Selection}

Prior to the video editing, a graphic overlay representing the inside of a fire appliance was created (Figure 1). To prevent the graphic overlay from obscuring the forwards view, the A-pillars and internal roof were created to be semi-transparent. This was done in an effort to mimic the effects of stereopsis and head movements, which naturally minimise A-pillar obscuration in real driving. The footage from the 7 camera streams was synchronised and edited in Adobe Premiere Pro CC. A team of traffic and transport psychologists, and fire service drivers and trainers, reviewed the footage in order to select the most promising stimuli. A total of 18 clips were chosen from the footage on the basis that each of these clips culminated in a narrow gap that the driver of the fire appliance at the time of filming either navigated through ( 9 'go trials'), or stopped and waited for a larger gap to open ( 9 'no-go trials'). Gaps could be between two vehicles (including parked vehicles), or between a vehicle and road furniture. These clips stopped immediately prior to the film-driver making the decision to either go through the aperture or stop. Each clip would pause at this point (for 1 second). Following this a question mark appeared on the frozen screen, identifying the gap to be judged. The question-mark screen was visible for a further second, simply to ensure that participants were judging the intended aperture. Following this two-second pause, the screen was replaced by the first of two rating scales. Participants gave a rating of how likely they thought the fire appliance would fit through the gap by selecting an appropriate radio button with the computer mouse (the 'will-it-fit?' scale, ranging from 1-8 with 8 being 'definitely fit'). The second scale asked participants to rate of how confident they would be to 
proceed through the gap at this point in time (the 'confidence-to-proceed' scale, ranging from 1-8 with 8 being 'extremely confident'). See Figure 1 for an example of a gap judgement clip.

\section{Data collection apparatus}

The test was presented on a computer monitor, measuring $48.3 \mathrm{~cm} \times 30.5 \mathrm{~cm}$. The monitor was connected to a SensoMotoric Instruments' Remote Eye-tracking Device, sampling at $500 \mathrm{~Hz}$ (SMI RED 500) with a $50 \mathrm{~ms}$ threshold for fixations. A high-speed event detection algorithm used a maximum peak velocity threshold of $40 \% \mathrm{~s}$ to identify saccades. Participants were provided with a mouse to select their answers.

\section{Questionnaires}

Participants were first asked to complete a small battery of questionnaires including: demographics, driving and crash history, and the Driver Behaviour Questionnaire (Short Version, Parker et al., 1995). We also gave participants the Traffic Locus of Control Questionnaire (T-LOC; Özkan \& Lajunen, 2005), the Sensation seeking scale (SSS; Zuckerman 1964) and a questionnaire regarding attitudes towards hazard perception. However, none of these produced any significant results and will not be discussed further.

The Driver Behaviour Questionnaire (DBQ; Reason et al., 1990; Parker et al., 1995) asks drivers to rate their frequency of certain behaviours while driving that are likely to increase the risk of collision. Its primary distinction is between errors (failures of planned actions to achieve their intended goal) and violations (deliberate acts that increase risk and decrease safety). A third set of questions targets lessor errors, termed 'slips and lapses'. The popularity of this questionnaire is evidenced in over 200 published studies (Winter \& Dodou, 2010), where it is often used to predict crash risk. 


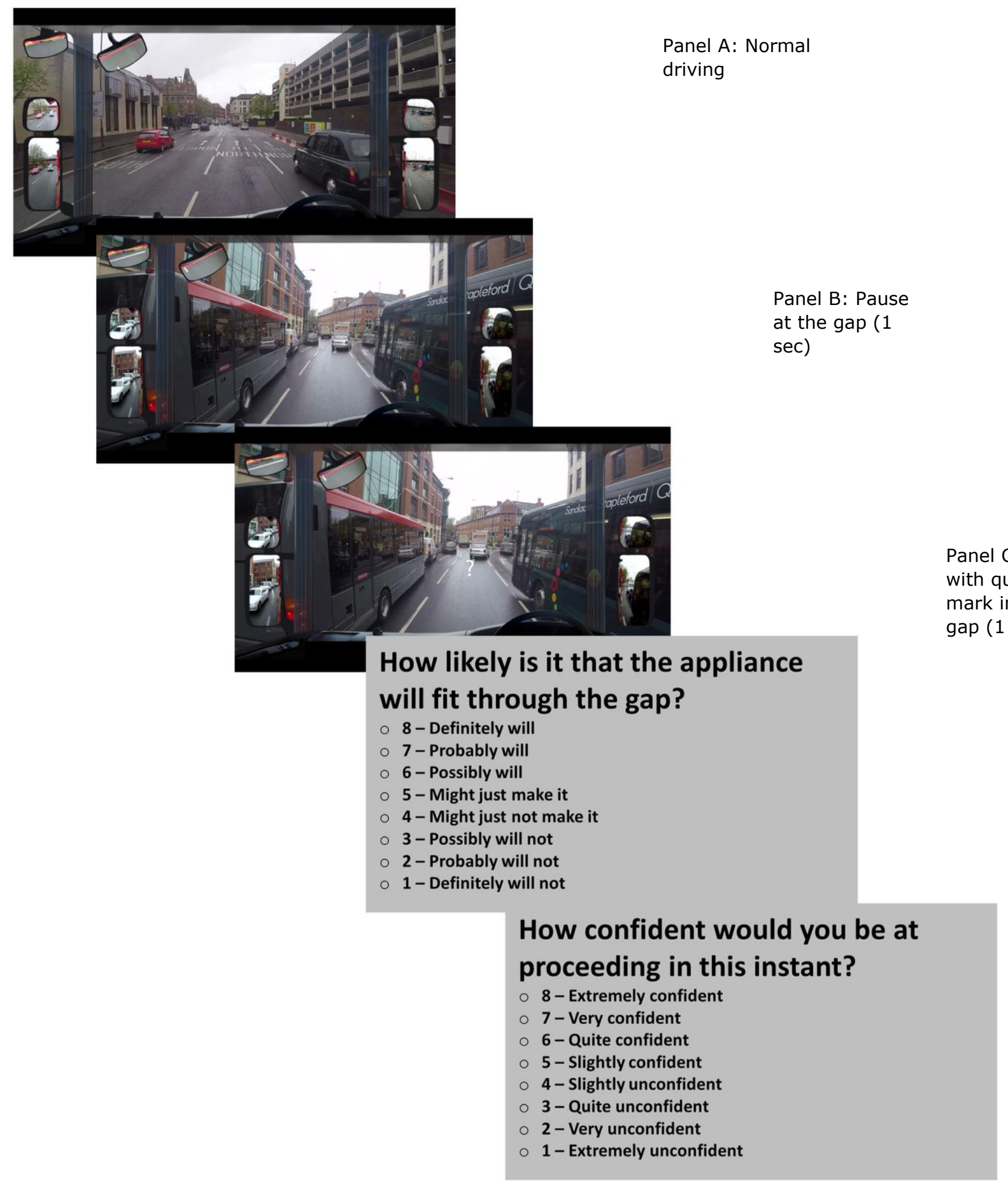

Figure 1. This Figure depicts a typical Gap Judgement clip. Panel A shows normal driving before a gap. Panel B shows the pause at the end of the video clip (1 second) which is quickly followed by Panel $\mathrm{C}$, where a question mark reinforces which gap the participant needs to judge (1 second). Panel D shows the 'will-it-fit?' rating. Panel E shows the 'confidence-to-proceed' rating. 


\section{Design}

A 2 × 4 mixed design was used with two types of clips ('go' and 'no-go' clips) and four driver groups: control drivers, novice FA drivers, high-risk, experienced FA drivers, and low-risk, experienced FA drivers. All participants watched all 18 gap judgement clips, presented in a random order. The main dependent variables were participants' ratings regarding whether they believed the appliance would fit through the gap, and how confident they would be to drive through that gap themselves (on a 1-8 scale, with higher numbers reflecting a greater belief that it will fit, and greater confidence).

Additional dependent variables included a selection of eye movement measures that were recorded to reflect the amount of attention that participants gave to the apertures in the clips. These included mean fixation durations; net dwell time and the number of fixations on 3 Areas of Interest (AOls; see Figure 2 for example of the AOI's). The AOls for the gap judgement test were on the left, centre, and right of the upcoming gap (mean coverage for each AOI across all clips was $0.83 \%$ (of the visual scene; range between $0.2 \%-2 \%$ ). The central $\mathrm{AOI}$ represented the roadway through the gap, while the left and right AOIs represented the objects that defined the gap (e.g. a parked car on the left, and a bus on the right), these AOIs were always the same size in each clip. Fixations on these AOIs were recorded for one second leading up to the point at which the video froze, to capture the cues that drivers were using to judge whether their 'vehicle' would fit through. This one second analysis window ensures that these eye movements are relevant to the gap. Prior to one second, the variation in stimuli makes comparison of eye movements difficult across clips. A series of $2 \times 4$ Analyses of Variance (ANOVAs) comparing across the four participant groups was used to analyse these measures. Where appropriate, a multilevel binomial Generalised Linear Model was used for the accuracy data.

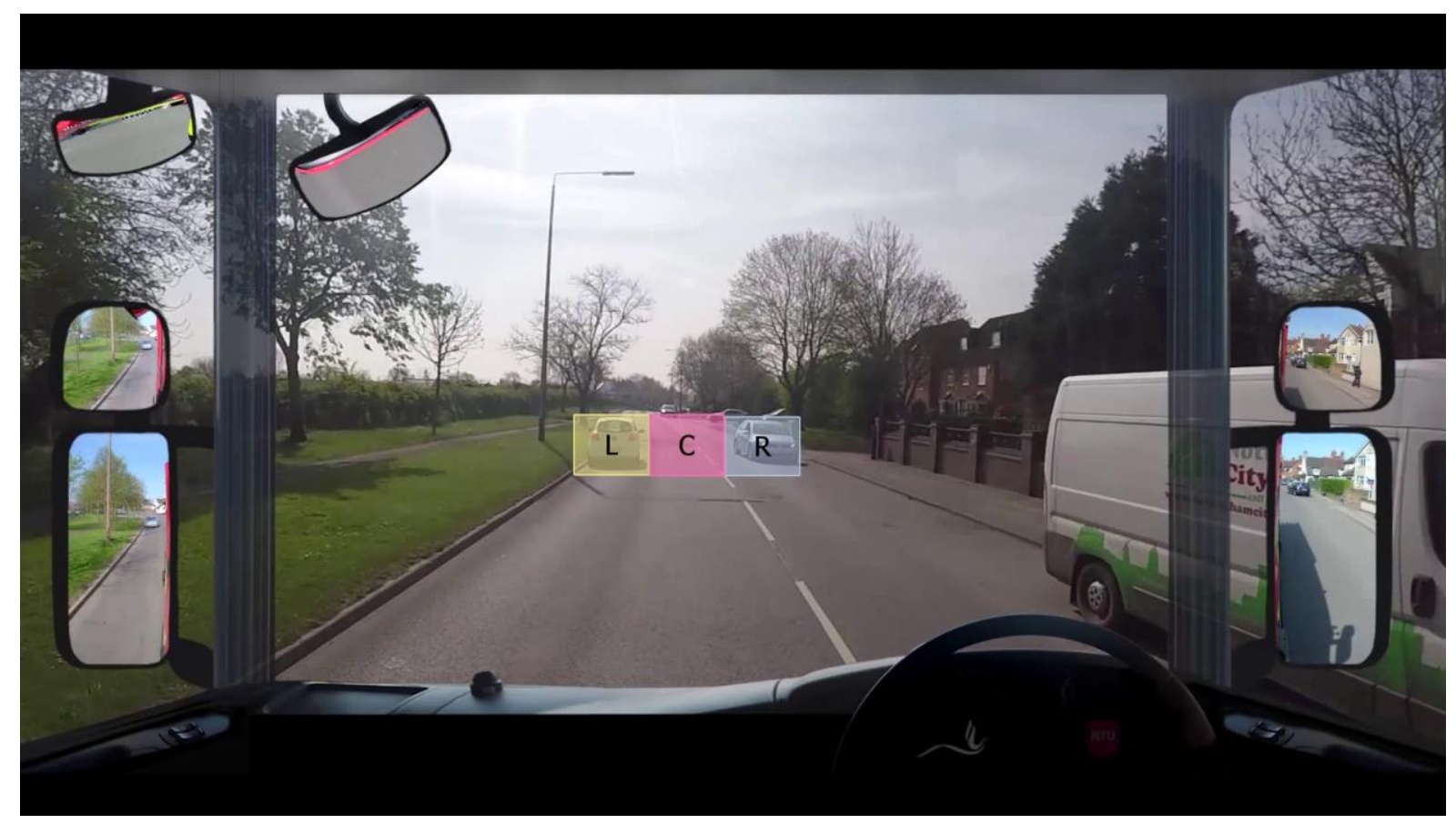

Figure 2. This Figure depicts the areas of interest for a typical go-trial. Left AOI denoted by the letter ' $\mathrm{L}$ ', central AOI denoted by the letter ' $C$ ' and the right AOI denoted by the letter ' $R$ '. 


\section{Procedure}

All Fire Service personnel gained permission from their respective watch managers and completed the test in a quiet office in their respective Nottinghamshire fire stations while on shift. Control participants were tested in an eye-tracking laboratory at Nottingham Trent University. Participants were first asked to complete a battery of questionnaires including: demographics, driving and crash history, the Driver Behaviour Questionnaire (Short Version, Parker et al., 1995), Traffic Locus of Control Questionnaire (T-LOC; Özkan \& Lajunen, 2005), Sensation Seeking (SSS; Zuckerman 1964) and attitudes towards hazard perception. Participants were informed that the questionnaires were completely anonymous and that they did not have to answer any of the questions if they did not want to.

In total participants completed three tests: the gap judgement (experiment 1), a hazard perception test (experiment 2) and a hazard prediction or 'what happens next?' test (experiment 3;

(for further details on experiments 2 and 3 please see Crundall \& Kroll, 2018). The order of the perception and prediction tests was counterbalanced, and they were presented either before or after the gap judgement task. As a check, the order of the gap test and the other two experiments was included as a factor in all analyses, but was not found to have an effect.

Participants were seated at approximately $60 \mathrm{~cm}$ away from the screen for all tests. They were told that the videos clips that they were about to watch were taken from the perspective of a FA driver on an emergency response drive (i.e., a blue-light run).

Participants were instructed to watch the clips looking for gaps through which the fire appliance would have to negotiate. They were informed that each clip would pause on approach to the gap. Following a one-second pause, a question mark symbol appeared to indicate the gap in question. This lasted for a further second before participants were presented with the 'will-it-fit?' rating scale (from 1 to 8). Following their mouse-button response, they were then presented with the 'confidence-to-proceed' scale (from 1 to 8 ), again requiring a mouse-button response.

\section{Results}

The main measures of the gap judgement test were participants' ratings regarding whether they believed the appliance would fit through the gap, and how confident they would be to drive through that gap themselves (on a 1-8 scale, with higher numbers reflecting a greater belief that it will fit, and greater confidence). Additional analyses compared questionnaire and eye movement data across the driver group using Analyses of Variance (ANOVA). Following this a series of planned Helmert contrasts were undertaken comparing (1) control drivers to all FA drivers, (2) novice FA drivers to all experienced FA drivers, and (3) high-risk, experienced FA drivers to low-risk, experienced FA drivers. One participant was removed (a low-risk, experienced driver) due to poor performance on the hazard perception test, which suggests that they were not sufficient engaged in the research. Another participant (a novice FA driver) was also removed from the analysis due to loss of data.

\section{Gap ratings}

'Will-it-fit?' Ratings

A $2 \times 4$ mixed ANOVA compared the average ratings for the 'will-it-fit' scale across the four participant groups, for both 'go clips' and 'no-go clips'. This revealed a significant main effect of go/no-go status, $F(1,78)=455.42, M S E=0.4, p<0.001$, confirming that participants rated the gap in 'go clips' as more 
likely to accommodate the fire appliance than 'no-go clips' (6.67 vs. 4.57, respectively). While there was no main effect of driver group, the interaction between go/no-go clips and driver group approached significance, $F(1,78)=2.41, M S E=0.4, p=0.07)$. As can be seen in Figure 3 , the different driver groups gave similar ratings for the 'go clips' (all $p$ 's $<0.05$ ). For the 'no-go clips' however the novice fire-appliance drivers appear more likely to believe that the vehicle will fit through the gap than other the other groups.

\section{'Will-It-Fit' Accuracy}

While the mean ratings reflect fine distinctions in drivers' beliefs regarding the relationship between the aperture and the fire appliance, they do not consider the accuracy of their response. It was made clear to participants that a rating of 5 or above represented a 'go' decision while a rating of 4 or below reflected gradations of a 'no-go' decision. Accordingly, participant responses were recoded into 'go' and 'no-go' responses, and were then compared to the actual behaviour of the film-driver. The number of correct responses were calculated for each participant for go and no-go trials, and were analysed as a multilevel binomial Generalised Linear Model. The main effects model identified a significant effect of go/no-go trials (via dropping a level of go/no-go trials and comparing to the full main effects model; $\left.\chi^{2}(1)=319, p<.001\right)$. Though no such effect was found for driver group $\left(\chi^{2}(3)=\right.$ $5.22, p=.16)$, the Helmert contrasts showed that novice drivers performed significantly worse than the two experienced groups of appliance drivers (63.6\% vs. $69.4 \%$, respectively; $Z=-2.287, p=.02$ ). See Figure 4 for the means. The interaction between go and no-go trials with driver group did not account for any more variance than the main effects model $\left(\chi^{2}(3)=5.56, p=0.14\right)$. Measures of sensitivity and criterion were also calculated following Signal Detection Theory (Stanislaw \& Todorov, 1999). These measures did not add to the interpretation of these results however: sensitivity followed the pattern seen in Figure 4, while criterion did not differ across groups.

\section{'Confidence-to-proceed' Ratings}

Participants' ratings of how confident they would be to proceed through the aperture at that point in time (i.e. at the point of the pause) were analysed via a $2 \times 4$ mixed ANOVA across 'go/no-go' clips and the four driver groups. This revealed a significant main effect of go/no-go clips, $F(1,78)=293.44, M S E$ $=0.45, p<0.001$, confirming that participants were significantly more confident in proceeding through the gaps presented in 'go clips' than 'no-go clips' (6.3 vs. 4.5). There was no significant main effect of driver group, $F(3,78)=0.31, M S E=0.92, p=0.82$. However, there was a significant interaction between driver group and go/no-go status, $F(3,78)=2.70, M S E=0.45, p=0.05$. As can be seen in Figure 5, while confidence remains stable across all driver groups on the 'no-go' clips, confidence regarding the 'go clips' improves across the four groups (i.e. gets closer to a rating of 8), with the most confident drivers being the low-risk, experienced fire-appliance drivers.

\section{Questionnaire data}

Participants' responses across the 24 DBQ items were averaged for the three factors (with 8 questions typically loading on errors, violations and slips/lapses). Cronbach's alpha for all three was acceptable $(0.83,0.73,0.66$, respectively). The resultant participant means for the three factors were entered into a series of $1 \times 4$ ANOVAs. In all factors, the high-risk experienced fire-appliance drivers scored higher in errors, violations and slips/lapses than the low risk group. As the questionnaire data was common to both the current study and Crundall and Kroll (2018), we refer the reader to the previous publication for more details of this analysis. 


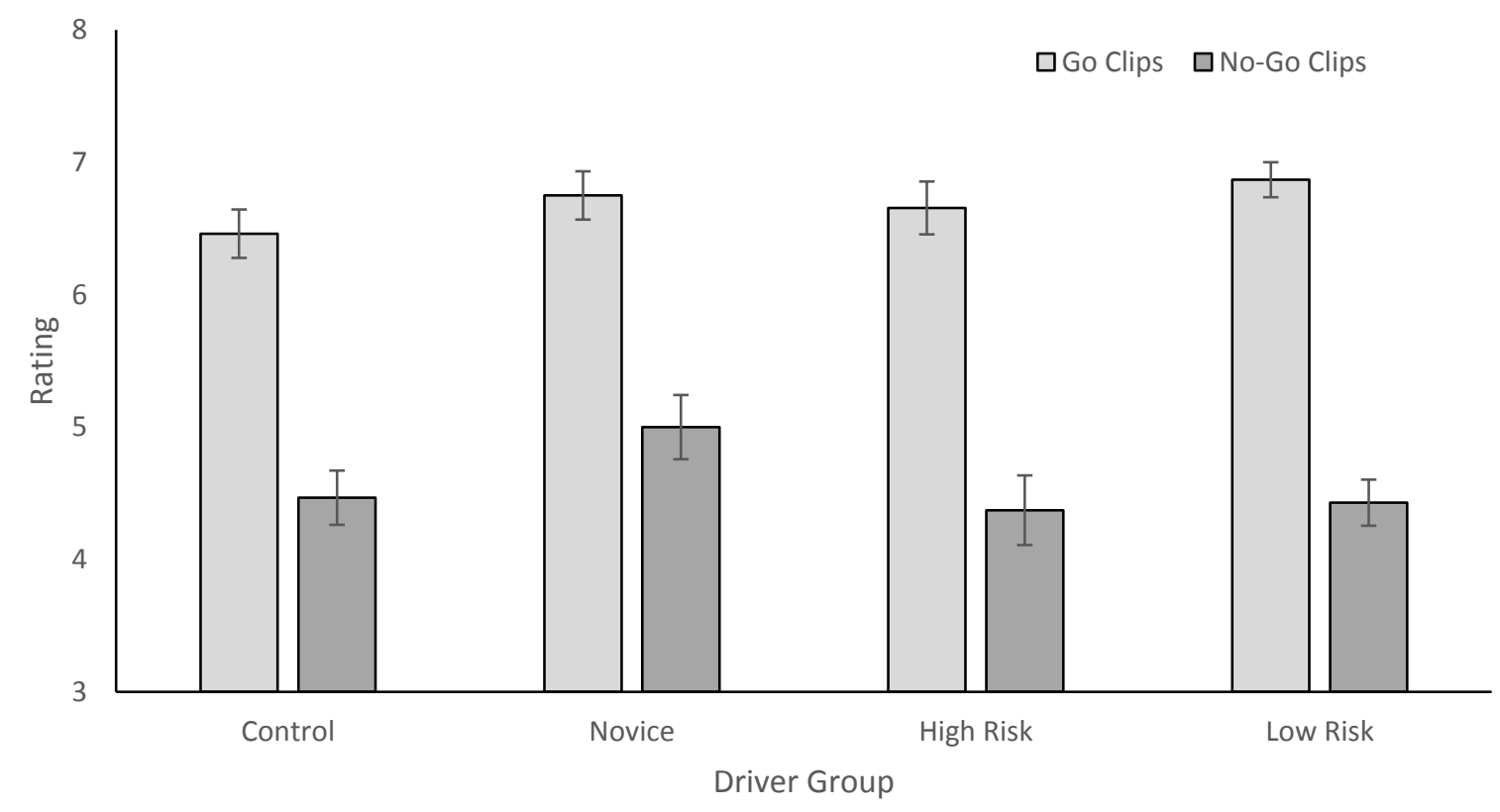

Figure 3. 'Will-it-fit?' ratings across the four driver groups for 'go' and 'no-go' clips. Ratings were given on an eight-point scale, with higher numbers reflecting a belief that the vehicle will fit through the gap. Standard error bars are included.

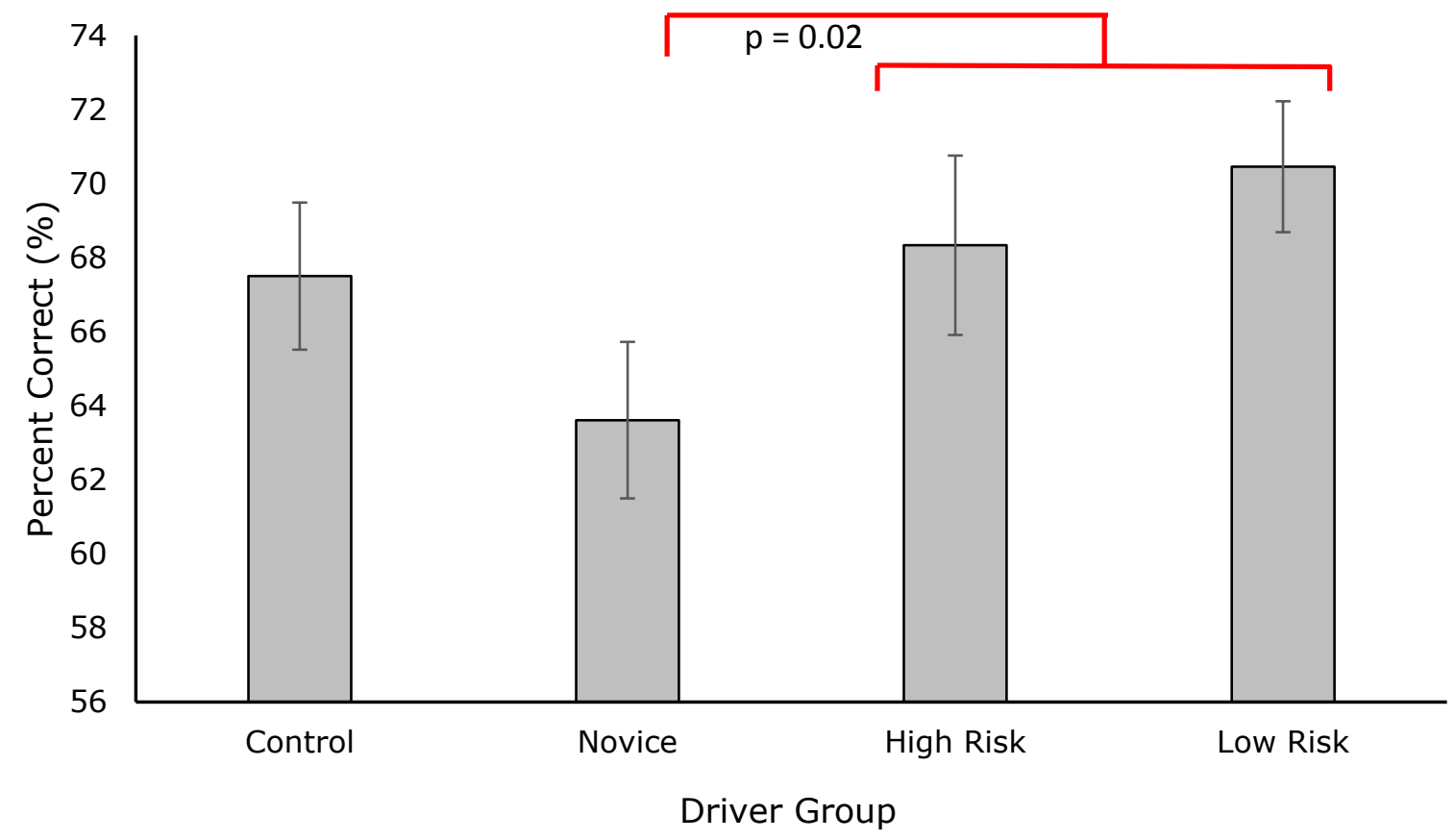

Figure 4. The percentage of clips across the different driving groups where participants correctly identified whether the appliance would fit through the gap or not. Standard error bars are included. 


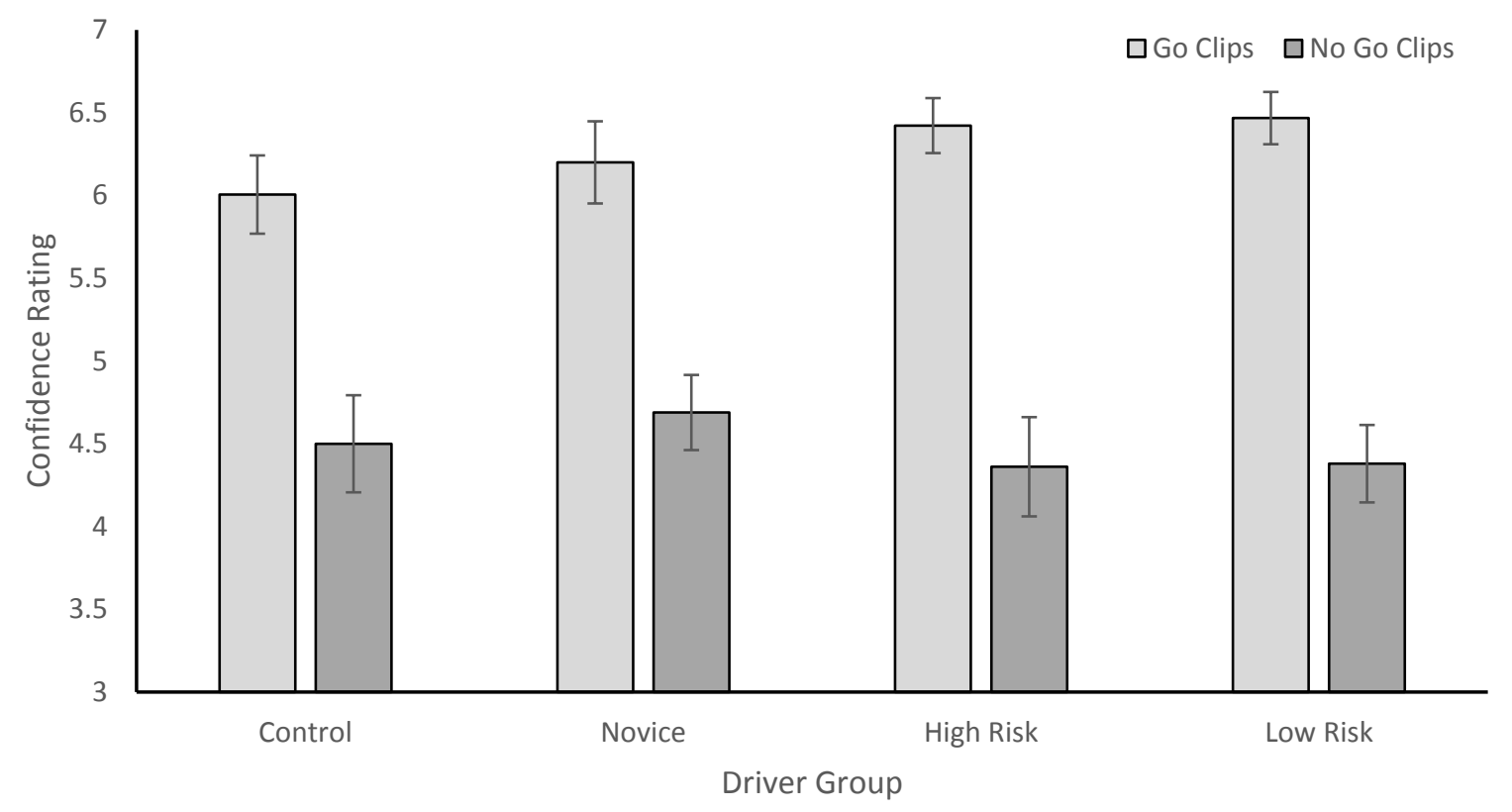

Figure 5. Confidence ratings for each clip type across all levels of driving experience. Ratings were given on an eight-point scale, with higher numbers reflecting a higher confidence rating. Standard error bars are included.

\section{Analysis of Eye Movements}

\section{Amount of Attention Devoted to the Aperture}

Several measures were recorded to reflect the amount of attention that participants gave to the apertures in the clips. These included mean fixation durations, net dwell time and the number of fixations on 3 Areas of Interest (AOIs). The AOIs for the test were on the left, centre, and right of the upcoming gap, for 1 second before the video paused. The central AOI represented the roadway through the gap, while the left and right AOls represented the objects that defined the aperture (e.g. a parked car on the left, and a bus on the right). Fixations on these AOIs were recorded for one second leading up to the gap, to capture the cues that drivers were using to judge whether their 'vehicle' would fit through.

\section{Mean Fixation Durations}

Participants' mean fixation durations were calculated for the three AOIs (left, centre, right). This measure is normally associated with cognitive load (Jacob \& Karn, 2003), with longer fixations indicating a longer processing time than shorter fixations. Novice drivers have been found to have longer fixations on hazards than experienced drivers, presumably reflecting increased processing demands due to their inexperience with interpreting such situations (Chapman and Underwood, 1998). A mixed $2 \times 3 \times 4$ ANOVA compared mean fixation durations across go/no-go clips, 3 AOls (left, centre and right) and the four driver groups.

This analysis revealed a main effect of go/no-go trials, $F(1,75)=5.38, M S E=8186.7, p=0.02$, with 'go clips' evoking longer fixations overall compared to the 'no-go clips' (295 ms vs. $276 \mathrm{~ms}$ ). There was 
also a significant main effect of driver group, $F(1,3)=2.98, M S E=20601.63, p=0.04$. Planned Helmert contrasts revealed that novice fire-appliance drivers had significantly longer fixations than the experienced fire-appliance drivers (316 ms vs. $269 \mathrm{~ms} ; p=0.01$ ).

There was also a significant interaction between $\mathrm{AOI}$ location and driver group, $F(6,150)=2.11, M S E$ $=7266.13, p=0.05$. As can be seen from Figure 6 , fixation durations on the left AOI are very similar across all groups. While fixations on the central AOI are ostensibly shorter for the two experienced fire-appliance groups there are no significant differences here either (all $p s>0.05$ ). However, posthoc $t$-test comparisons identified that novice drivers had significantly longer fixations on the right $A O I$ than all other groups (all ps $<0.05$ ). It is especially noticeable that the greatest difference in fixation length on the right edge of the aperture is found between novice drivers (with relatively long fixations) and low-risk experienced drivers (with exceptionally short fixations).

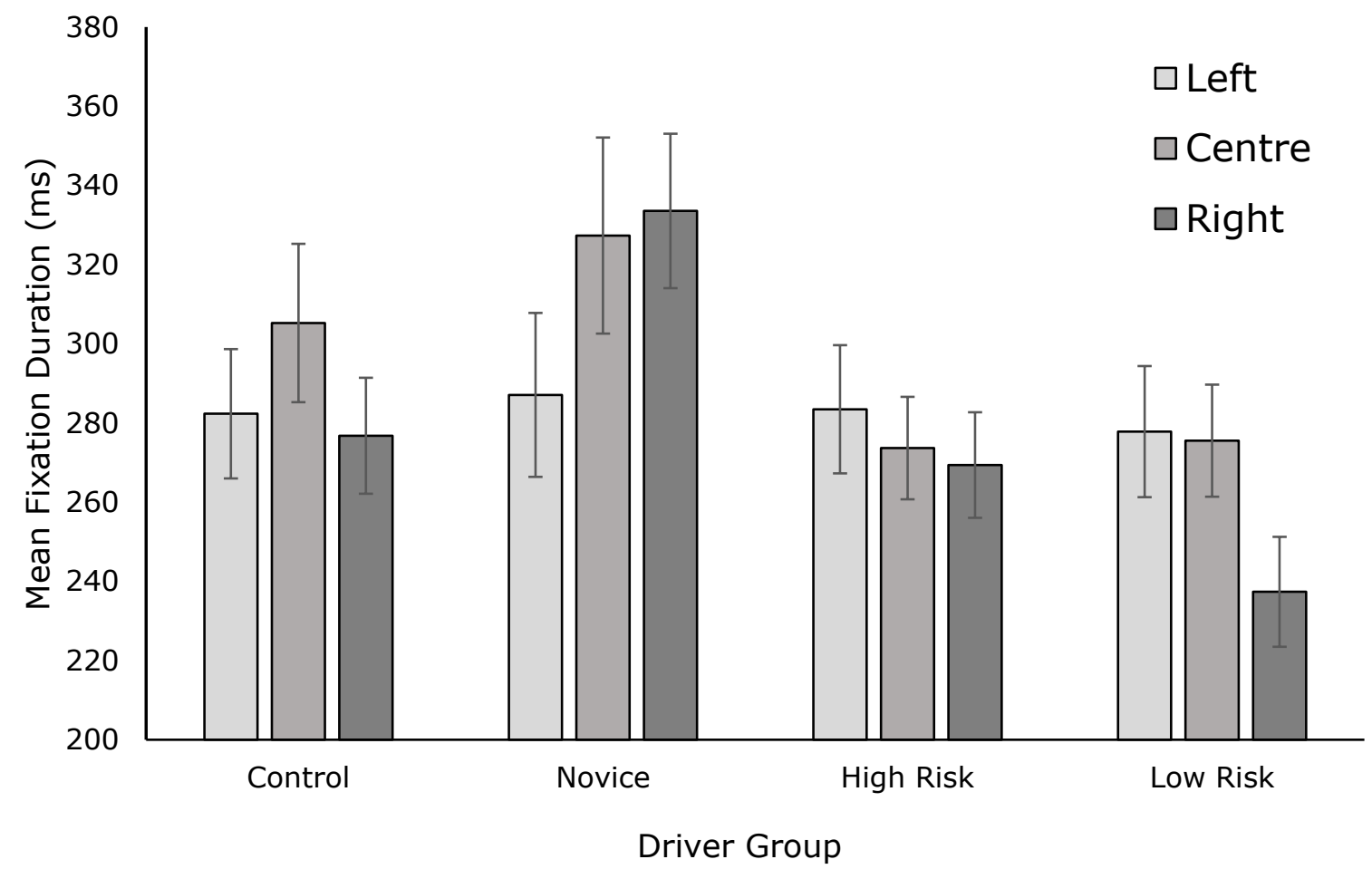

Figure 6. Mean fixation duration for each Driver Group for each AOI location (the left edge of the gap, the roadway through the centre of the gap, and the right edge of the gap). Standard error bars are included.

\section{Total Dwell Time}

For the current analyses, the measure of dwell time was chosen to reflect attention given to AOIs on the forthcoming gap prior to the video pausing. The dwell-time measure was calculated as the sum of all eye tracking samples that fell on these AOIs in the one second time window immediately preceding the pause of the video. A mixed 2 (go clips vs. no-go clips) $\times 3$ (AOI location: left, centre, right) $\times 4$ (driver group: control, novice, high-risk, low-risk) ANOVA was conducted on these data. While several interesting effects were noted (e.g. all drivers tend to favour the centre of the aperture and the right bounding edge, more so than the left edge), none of these discriminated between the driver groups and are therefore not discussed further. 


\section{Number of Fixations on the AOI's}

The number of fixations each participant made on the three AOIs (left, centre, right) were calculated. A mixed 2 (go vs. no-go) $\times 3$ (AOI location: left, centre, right) $\times 4$ (driver group: control, novice, highrisk, low-risk) ANOVA was conducted on these data. While several interesting effects were noted (e.g. once again, all drivers made more fixations on the centre of the gap, and the right edge of the gap, more so than the left edge of the gap), none of these discriminated between the driver groups. There was, however, a marginally significant effect of driver group, $F(3,75)=2.58, \mathrm{MSE}=0.31 p=0.06$, reflecting that novices drivers $(M=2.33)$ made more fixations within all three categories overall than all other driver groups (control $=2.07$, low-risk $=2.07$, high-risk $=2.10$, all $p^{\prime} s<0.05$ ). This suggests that the experienced FA drivers were extracting gap judgement information from the AOI's with fewer fixations than the novices, leaving the experienced drivers able to look at other areas in the scene.

\section{Discussion}

The aim of this study was to create a test that could successfully discriminate between driver groups in terms of their ability to accurately judge whether a FA would fit through a gap between parked vehicles or roadside furniture. This was done to address a common form of collision in the fire-service involving low-speed manoeuvres, where drivers try to navigate through apertures that are too small for their vehicle. Although this type of collision might be less life-threatening than the result of a failure in hazard perception (see Crundall and Kroll, 2018), they are costly for the fire-service in terms of both financial losses and public faith.

First, we predicted that all FA drivers would be more accurate at judging the gap than control drivers. Contrary to expectation, the test failed to demonstrate a distinction between FA drivers and controls. Second, we hypothesised that experienced FA drivers would outperform the novice FA drivers on the test. In keeping with this hypothesis, the results successfully differentiated novice FA drivers from their more experienced counterparts. The novices were significantly less accurate in deciding whether the appliance would fit through the gaps, and there is evidence from the eye movement data that this may be linked to a preoccupation with whatever object defined the right side of the gap. It was noticeable in the analysis of total dwell time that all participants favoured the right edge of the gap over the left edge, but the analysis of mean fixation durations suggests that this was a particular issue for the novice drivers. Third, we predicted that low-risk experienced FA drivers would perform better than high-risk FA drivers. However, no differences were observed between these two groups.

The behavioural distinction between the novice FA drivers and experienced FA drivers in is line with the limited number of studies that suggest novice drivers to have a greater difficultly with gap acceptance than experienced drivers (Clarke, 2000; Crinson \& Grayson, 2005; Forsyth, 1995; Clarke et al., 1998; Forsyth, 1995; West \& French, 1993; Scott et al., 2013; Crundall et al., 2012). However, a marked difference between these studies and the current study is that the former examined whether the gap in a flow of traffic at a junction is sufficient to pull out, whereas the current study looked at an aperture judgement between two vehicles or road furniture. To the authors' knowledge, no other study has looked at this variant of gap-judgement task in any vehicle; the current study therefore represents a novel paradigm for exploring this skill. The difference between novice and experienced groups is particularly striking considering that the drivers are not physically sat in a FA when making these judgements, and the absolute distance of the gap in the video (as it impacts on the retina) is less than it would be in real life. Both potential limitations could have restricted the ability of the video- 
based test to tap into real-world underlying skills. Despite these potential confounds, the test has demonstrated some success.

The bias towards attending to the right edge of the gap over the left edge is particularly interesting and worth noting. This bias may reflect the saliency of the right edge to drivers, i.e., It is the edge of the aperture that is closest to the driver in a right-hand vehicle, thus it may be a natural response to focus upon this edge due to its behavioural urgency (i.e. we look at it because it is the most likely object to cause us personal harm; Franconeri \& Simons, 2003). The camera that recorded the forward view was placed centrally on the FA windscreen and therefore did not favour right edges over left edges (as a true driver's perspective might). Thus, any argument for behavioural urgency leading to a bias for right edges must be based on learned associations rather than pure visual salience.

Alternatively, this right-edge bias may reflect a distinction between the types of objects that define both sides of the gap. Vehicles that define the left edge of a gap are more likely to be facing away from the FA, whereas right-edge vehicles may face towards the FA. This may create a saliency bias. Furthermore, any oncoming traffic might typically be considered more dangerous as any speed differential is likely to be greater than that of vehicles on the left. FAs also often depart their lane to avoid cars and road furniture on the left, putting them at further risk of a collision with oncoming traffic, making the right side of the road potentially more dangerous.

Nonetheless, regardless of the reason for a greater proportion of attention being devoted to the right edge rather than the left edge, the longer fixation durations of the novice drivers suggest that they are overly concerned with this gap cue, or are having greater difficulty processing it (Chapman \& Underwood, 1998; Crundall \& Underwood, 1998; Mourant \& Rockwell, 1972). This raises the possibility that aperture-judgement training might improve novice drivers' assessment of whether they can fit an appliance through a gap. It is too early to suggest what type of training might be most appropriate, but we now have a validated assessment tool to tell whether any targeted training interventions improve novices' aperture judgements.

As an interesting aside, it is worth noting that novices' accuracy performance on the test (Figure 4) appears to be even worse than the control drivers, which would suggest that the novices appear to have taken a retrograde step in their judgement abilities. The novice group was not identified as being particularly error-prone, or likely to commit violations, suggesting that this error may be very specific to this type of task. It is possible that this error may arise out of initial HGV or FA training. For instance, one might imagine that drivers who have never driven an HGV or fire appliance (the control group) would base their decision simply on what they can see, coupled with a naïve understanding of the width of an appliance. The fact that novice drivers appear to be worse than control drivers suggests that they are adding erroneous information to their decision-making process. This may reflect their early attempts to integrate the fire appliance into their body schema, adding to their peripersonal space. At early stages of expanding peripersonal space through experience and training, novice drivers may require negative feedback to calibrate their integration with the vehicle (i.e. performance may need to get worse before it gets better). If this explanation is true, then there is an argument for additional training in handling-skills, perhaps using soft objects to define gaps for novices to navigate through on a training ground.

These results have identified a potentially dangerous period for new FA drivers regarding decisions to navigate through apertures. One caveat should be mentioned however. While the appearance of hazards on a video clip may be a good representation of the same hazard in real life, an aperture in traffic is a lot harder to portray in video. Participants watching the clips are not sat within the reference frame that must be compared to the gap (i.e. they are not sat in a fire appliance). Even if they were, 
the gap portrayed in the video is not the real size as that encountered in real life, but is only indicative in terms that are relative to the context. It is possible that successful aperture judgements are based on an internal representation of how far the eyes jump from one edge of the gap to the other, which is then compared to one's understanding of the width of the vehicle (perhaps encoded in terms of peripersonal space). If that is the case, the smaller absolute size of the gap in the video clips may cause problems as one might end up comparing a tiny video-representation of a gap to the real width of a fire appliance. The fact that the current test differentiates between the groups following our predicted pattern (i.e. novices perform worse than experienced drivers) suggests that this is not the case, though it would be prudent to compare performance on the aperture judgement test to real world estimations of gaps.

In conclusion, this study represents the first attempt to develop an aperture-judgement test specifically for fire-appliance drivers on blue-light runs. The test has successfully demonstrated the ability to differentiate between novice fire-appliance drivers and experienced fire-appliance drivers, with the latter outperforming the former. This suggests that the test successfully taps into experienced FA drivers' specific skill set of aperture judgement. This is even more impressive given that participants were not actually sat in an appliance making these judgements. The success of this test can be used to form the basis of a future training and assessment tool for fire-appliance drivers in the hope of reducing the occurrence of some of the collisions that occur due to failure in aperture judgement, whilst allowing training resources to be better targeted in the fire service.

\section{Acknowledgements}

This work was supported by The Fire Service Research and Training Trust, UK [2015-16]. We would like thank Craig Parkin, Paul Foster, Ivan Hayes, Vivienne Brunsden and Mark Jarman for all of their assistance, and all of the emergency response drivers (and control participants) who gave up their time to participate.

\section{References}

Alexander, J., Barham, P., \& Black, I. (2002). Factors influencing the probability of an incident at a junction: Results from an interactive driving simulator doi:https://doi.org/10.1016/S0001$\underline{4575(01) 00078-1}$

Berthelon, C., \& Mestre, D. (1993). Curvilinear approach to an intersection and visual detection of a collision. Human Factors, 35(3), 521-534.

Bottom, C., \& Ashworth, R. (1978). Factors affecting the variability of driver gap-acceptance behaviour. Ergonomics, 21(9), 721-734.

Caird, J., \& Hancock, P. (1994). The perception of arrival time for different oncoming vehicles at an intersection. Ecological Psychology, 6(2), 83-109. 
Chapman, P. R., \& Underwood, G. (1998). Visual search of driving situations: Danger and experience. Perception, 27(8), 951-964.

Clarke, D. (2000) Analysis of Novice Driver Accidents, University of Nottingham. DETR Novice Drivers Conference Proceedings.

Clarke, D. D., Forsyth, R., \& Wright, R. (1998). Behavioural factors in accidents at road junctions: The use of a genetic algorithm to extract descriptive rules from police case files. Accident Analysis \& Prevention, 30(2), 223-234.

Crinson, L. F., \& Grayson, G. B. (2005). Profile of the british learner driver. Traffic and Transport Psychology, Theory and Application, , 157-170.

Crundall, D., \& Kroll, V. (2018). Prediction and perception of hazards in professional drivers: Does hazard perception skill differ between safe and less-safe fire-appliance drivers? Accident Analysis \& Prevention, 121, 335-346.

Crundall, D. E., \& Underwood, G. (1998). Effects of experience and processing demands on visual information acquisition in drivers. Ergonomics, 41(4), 448-458.

Crundall, D., Chapman, P., France, E., Underwood, G., \& Phelps, N. (2005). What attracts attention during police pursuit driving? Applied Cognitive Psychology, 19(4), 409-420.

Crundall, D., Chapman, P., Phelps, N., \& Underwood, G. (2003). Eye movements and hazard perception in police pursuit and emergency response driving. Journal of Experimental Psychology: Applied, 9(3), 163.

Crundall, D., Crundall, E., Clarke, D., \& Shahar, A. (2012). Why do car drivers fail to give way to motorcycles at t-junctions? Accident Analysis \& Prevention, 44(1), 88-96.

DeLucia, P. R. (1991). Pictorial and motion-based information for depth perception. Journal of Experimental Psychology: Human Perception and Performance, 17(3), 738-748.

Dewing, W., Duley, J., \& Hancock, P. (1993). The role of vehicle type, velocity and gap size on driver left-turn decisions. 37th Annual Meeting of the Human Factor Society, Seattle, WA, 
Du, W., Wilmut, K., \& Barnett, A. L. (2015). Level walking in adults with and without developmental coordination disorder: An analysis of movement variability. Human Movement Science, 43, 9-14.

Forsyth, E. (1995). Cohort Study of Learner and Novice Drivers: Part 3, Accidents. Offenses and Driving Experience in the First Three Years of Driving. TRL Project Report 111.

Franchak, J. M., \& Adolph, K. E. (2012). What infants know and what they do: Perceiving possibilities for walking through openings. Developmental Psychology, 48(5), 1254.

Franchak, J., \& Adolph, K. (2014). Affordances as probabilistic functions: Implications for development, perception, and decisions for action. Ecological Psychology, 26(1-2), 109-124.

Franconeri, S. L., \& Simons, D. J. (2003). Moving and looming stimuli capture attention. Perception \& Psychophysics, 65(7), 999-1010.

Galli, G., Noel, J. P., Canzoneri, E., Blanke, O., \& Serino, A. (2015). The wheelchair as a full-body tool extending the peripersonal space. Frontiers in Psychology, 6, 639.

Hackney, A. L., Cinelli, M. E., \& Frank, J. S. (2015). Does the passability of apertures change when walking through human versus pole obstacles? Acta Psychologica, 162, 62-68.

Hackney, A. L., Cinelli, M. E., \& Frank, J. S. (2014). Is the critical point for aperture crossing adapted to the person-plus-object system? Journal of Motor Behavior, 46(5), 319-327.

Hancock, P., \& Caird, J. K. (1993). Experimental evaluation of a model of mental workload. Human Factors, 35(3), 413-429.

Hancock, P., Caird, J., Shekhar, S., \& Vercruyssen, M. (1991). Factors influencing drivers' left turn decisions. Proceedings of the Human Factors Society Annual Meeting, 35. (15) pp. 11391143.

Hancock, P., Caird, J., \& Johnson, S. (1991). The left-turn. In Proceedings of the International Ergonomics Association. Paris, France. 
Higuchi, T., Cinelli, M. E., Greig, M. A., \& Patla, A. E. (2006). Locomotion through apertures when wider space for locomotion is necessary: Adaptation to artificially altered bodily states. Experimental Brain Research, 175(1), 50-59.

Higuchi, T., Murai, G., Kijima, A., Seya, Y., Wagman, J. B., \& Imanaka, K. (2011). Athletic experience influences shoulder rotations when running through apertures. Human Movement Science, 30(3), 534-549.

Higuchi, T., Seya, Y., \& Imanaka, K. (2012). Rule for scaling shoulder rotation angles while walking through apertures. PloS One, 7(10), e48123.

Ho, C., \& Spence, C. (2009). Using peripersonal warning signals to orient a driver's gaze. Human Factors, 51(4), 539-556.

Holmes, N. P., Calvert, G. A., \& Spence, C. (2004). Extending or projecting peripersonal space with tools? multisensory interactions highlight only the distal and proximal ends of tools. Neuroscience Letters, 372(1-2), 62-67.

Horswill, M. S., Helman, S., Ardiles, P., \& Wann, J. P. (2005). Motorcycle accident risk could be inflated by a time to arrival illusion. Optometry and Vision Science, 82(8), 740-746.

Horswill, M. S., Taylor, K., Newnam, S., Wetton, M., \& Hill, A. (2013). Even highly experienced drivers benefit from a brief hazard perception training intervention. Accident Analysis \& Prevention, 52, 100-110.

Jacob, R. J., \& Karn, K. S. (2003). Eye tracking in human-computer interaction and usability research: Ready to deliver the promises. The mind's eye (pp. 573-605) Elsevier.

Johnston, K., \& Scialfa, C. (2016). Hazard perception in emergency medical service responders. Accident Analysis \& Prevention, 95, 91-96.

Karthika, P. T. \& Koshi, B. I. (2014) Gap Acceptance Behaviour of Drivers at T-Intersections. International Journal of Engineering Research \& Technology, vol.3, 11, pp 935 - 938.

Keskinen, E., Ota, H., \& Katila, A. (1998). Older drivers fail in intersections: Speed discrepancies between older and younger male drivers. Accident Analysis \& Prevention, 30(3), 323-330. 
Louveton, N., Bootsma, R. J., Guerin, P., Berthelon, C., \& Montagne, G. (2012). Intersection crossing considered as intercepting a moving traffic gap: Effects of task and environmental constraints. Acta Psychologica, 141(3), 287-294.

McKenna, F., \& Crick, J. (1991). Experience and expertise in hazard perception. Behavioural Research in Road Safety. Proceedings of a Seminar Held at Nottingham University, 26-27 September 1990, (PA 2038/91)

Mourant, R. R., \& Rockwell, T. H. (1972). Strategies of visual search by novice and experienced drivers. Human Factors, 14(4), 325-335.

Muroi, D., \& Higuchi, T. (2017). Walking through an aperture with visual information obtained at a distance. Experimental Brain Research, 235(1), 219-230.

Nagayama, Y., Morita, T., Miura, T., Watanabe, J., \& Murakami, N. (1980). Speed judgment of oncoming motorcycles. In Proceedings of international motorcycle safety conference (Vol. II, pp. 955-971).

Obaidat, T., \& Elayan, M. S. (2013). Gap acceptance behavior at U-turn median openings-case study in jordan. Jordan Journal of Civil Engineering, 7(3), 332-341.

Scott, H., Hall, L., Litchfield, D., \& Westwood, D. (2013). Visual information search in simulated junction negotiation: Gaze transitions of young novice, young experienced and older experienced drivers. Journal of Safety Research, 45, 111-116.

Stanislaw, H., \& Todorov, N. (1999). Calculation of signal detection theory measures. Behavior Research Methods, Instruments, \& Computers, 31(1), 137-149.

Warren Jr, W. H., \& Whang, S. (1987). Visual guidance of walking through apertures: Body-scaled information for affordances. Journal of Experimental Psychology: Human Perception and Performance, 13(3), 371.

West, R., French, D., Kemp, R., \& Elander, J. (1993). Direct observation of driving, self-reports of driver behaviour, and accident involvement. Ergonomics, 36(5), 557-567. 
